\title{
LOS HERBARIOS DE MEXICO, SU HISTORIA Y ESTADO ACTUAL
}

\author{
Carlos luis Díaz Luna* \\ Luz María Villarreal **
}

Agradecemos la honrosa invitación a participar en este Coloquio sobre los Herbarios de México, hecha por los doctores Jerzy Rzedowski y Gastón Guzmán, de esta Escuela Nacional de Ciencias Biológicas, del Instituto Politécnico Nacional. También estimamos la atención de las personas que respondieron a la solicitud de demanda de datos para formular este trabajo.

Al relatar la historia de los Herbarios mexicanos, sus colectores y estudiosos que han producido obras sobre plantas mexicanas no se puede cludir la referencia al famoso Herbario pictórico, conocido como "Códice Berberini" o "Manuscrito Badianus", escrito en lengua azteca en 1552 por Martín de la Cruz y traducido al latín por Juan Badiano, ambos indígenas, el primero de Thatelolco y el segundo de Xochimilco, en el que describen multitud de plantas así como sus virtudes medicinales.

Anuque estrictamente "Herbario" es una colección de plantas desecadas, catalogadas y arregladas para consulta, no se puede omitir tampoco la mención sobre las colecciones de plantas vivas, cultivadas por nuestros antepasados en hermosos jardines botánicos, que causaron la admiración y sorpresa de los conquistadores españoles, quienes también reconocieron el profundo conocimiento que los indígenas tenían sobre las plantas, mismos que condujeron a su vez a la domesticación y al mejoramiento de las que servían como alimento, como maíz, frijol, calabaza, cacao y muchas otras.

Netzahualcóyotl (1431-14.72) creó el Jardín Botánico de Teizcutzingo; Moctezuma I (1440-14.72) acrecentó el de Huastepec con plantas medicinales y de ornato; Cuitláhuac en 1520 plantó el Jardín Botánico de Ixtapalapan, des. tinado a plantas de tierra fría que no podían cultivarse en Huastepec. El de Chapultepec, bosque cultivado, fue fundado por Itzcóatl en 1453, con grandes árboles como el ahuehuete (Taxodium mucronatum), de los que aún quedan notables ejemplares y que fue lugar de recreo de los reyes mexicanos.

Igualmente se debe hacer mención de la monumental obra de Francisco

* Escuela de Biología, Universidad Autónoma de Guadalajara. Gudalajara, Jal.

** Escuela de Agricultura, Universidad de Guadalajara. Guadalajara, Jal. 
Hernández, con descripciones y dibujos de plantas, elaborada de 1570 a 1577 e intitulada "De Historia Plantarum Novae Hispaniae", en la que se hace referencia a más de 3000 especies.

El primer Herbario científico de México fue el de Mariano Mociño, de Temascaltepec, y Martín Sessé y Lacasta, español, quienes colectaron en compañía de Juan Diego de Castillo, Vicente Cervantes y José Longinos, bajo los auspicios de la Real Expedición Botánica entre 1787 y 1801. Exploraron aroplias áreas desde Sinaloa y Guanajuato hasta Veracruz, Tabasco y Chiapas. Este Herbario fue enviado a la colección del Jardín Botánico de Madrid. Mociño conservó 1,400 ilustraciones de plantas dibujadas a todo color, las cuales prestó a De Candolle, quien logró reproducir en 1817 la mayor parte de ellas y gracias a estas copias se conocen hoy los dibujos realizados por la Expedición. Los originales se perdieron. La Sociedad Mexicana de Historia Natural publicó en 1887 las obras de Mociño y Sessé "Flora mexicana" y "Plantae Novae Hispanie", en las cuales se describen alrededor de 1000 espicies.

Humboldt y Bonpland, en los postrimeríos de la época de la Colonia (1803-1804), visitaron gran parte de la Nueva España colectando copiosamente en su recorrido por los estados de Guerrero, Morelos, México, Hidalgo, Guanajuato, Querétaro, Michoacán, Puebla y Veracruz. Miles de ejemplares de Amado Bonpland se depositaron en el Herbario de París, mismos que fueron la base de sendos estudios en los cuales se dio a conocer un gran número de representantes de la flora de México.

En la época de la Independencia se multiplicaron las actividades de los colectores botánicos y tan sólo entre 1810 y 1855 cabe destacar los nombres de Lucas Alamán y su colaborador de apellido Méndez, Juan Martínez de Lexarza, Pablo de la Llave, Wilhelm Friedrich von Karwinski, Jean Louis Berlandier, Christian Julius Wilhelm Schiede, Thomas Coulter, G. Andrieux, Henri Galeotti, Auguste Ghiesbreght, Theodor Hartweg, Karl Ehrenberg, Frederik Michael Liebmann y Berthold Seemann.

No es, aparementemente, sino hasta la segunda mitad del siglo XIX cuando nacen los herbarios institucionales de la ciudad de México. Así se tiene noticia de colecciones depositadas en la Escuela de Agricultura, en la Escuela de Minas, en la Escuela Nacional Preparatoria, etc. En 1881 Manuel Urbina formalizó el herbario en el seno del Museo Nacional, que en 1887 contaba con unos 3000 ejemplares. En 1879 se fundó la Comisión Geográfica Exploradora y en 1888 el Instituto Médico Nacional y ambas instituciones entre sus actividades incluyeron la formación de colecciones de plantas. Sobre todo este último se destacó por su labor botánica a cargo de Fernando Altamirano, José Ramírez y Al- 


\section{HERBARIOS EN MEXICO}

fonso L. Herrera. Además del fruto de la actividad de diversos exploradores nacionales en esa época se adquirieron las importantes colecciones de Wilhelm Schaffner, de Cyrus G. Pringle y algunas de Edward Palmer. A la vuelta del siglo el herbario del Instituto Médico Nacional contaba con más de 9000 ejemplares identificados y debidamente arreglados.

En 1915 se fundó también en la ciudad de México el Instituto de Biología General y Médica, que poco tiempo después fue rebautizado como Dirección de Estudios Biológicos. Este organismo heredó el conjunto de los herbarios del Instituto Médico Nacional, del Museo Nacional y de la Comisión Geográfica Exploradora y entre sus principales botánicos cabe mencionar a Maximino Martínez y a Cassiano Conzatti, gracias a cuyos esfuerzos se han ido incrementando los acervos hasta llegar a más de 21,000 ejemplares en 1929, cuando la Dirección se transformó en el Instituto de Biología, dependiente de la Universidad Nacional Autónoma de México. Uno de los colectores más activos de esa época fue Jesús González Ortega, quien exploró porciones de Sinaloa, Nayarit y Durango.

Para 1937 este Herbario ya tenía 28,000 especímenes, prácticamente todos ellos de plantas vasculares, pero a partir de entonces comenzó también a fomentarse la parte criptogámica, merced a la labor de Manuel Ruiz Oronoz y posteriormente también de Teófilo Herrera y de otros más. Además de Maximino Martínez, también Helia Bravo, Faustino Miranda y Eizi Matuda han sido los botánicos principales en la sección de plantas vasculares entre 1940 y 1964. En 1968, gracias en gran parte a las cuantiosas donaciones de las colecciones de Matuda y de Miranda, el acervo total ascendió a más de 117,000 ejemplares.

De otros herbarios institucionales de la ciudad de México y de sus alrededores cabe mencionar el de la Escuela Nacional de Ciencias Biológicas del Instituto Politécnico Nacional, iniciado en 1943, el del Instituto Nacional de Investigaciones Forestales, fundado en 1958, y el de la Escuela Nacional de Agricultura, de Chapingo, Méx., cuyos orígenes se remontan a mediados del siglo pasado, pero que ha atravesado por numerosas altas y bajas y sólo ahora parece estar encaminado hacia una fase de recuperación definitiva.

En la provincia hubo varios intentos de formalizar herbarios en las primeras décadas del siglo, pero éstos en su mayoría no han logrado salir victoriosos de la prueba del tiempo. El caso más dramático quizás fue la reciente destrucción completa de la importante colección formada por Jesús González Ortega en Mazatlán.

En tiempos más recientes fueron creados los herbarios de la Universidad de Nuevo León, en 1942, del Instituto Tecnológico y de Estudios Superiores de 
Monterrey en 1948, del Instituto Botánico de Chiapas en 1949, de la Comisión Botánica del Gobierno del Estado de México en 1953, del Instituto de Investigación de Zonas Desérticas de la Universidad Autónoma de San Luis Potosí en 1954, de la Universidad de Guadalajara en 1960, de la Universidad Autónoma de Guadalajara en 1967 y de la Universidad Veracruzana en 1971.

A continuación se describen las características más sobresalientes de los principales herbarios activos en los momentos actuales.

\section{Herbario del Instituto de Biología de la Universidad Nacional Autónoma de México}

El Herbario del Instituto de Biología de la Universidad Nacional de México, también llamado Herbario Nacional, se puede considerar que fue fundado en el año 1881 por Manuel Urbina. Sus siglas son MEXU.

Su acervo actual asciende a 177,400 ejemplares, de los cuales 161,200 son fanerógamas, 9,000 hongos y mixomicetos, 4,000 pteridofitas, 3,000 briofitas y 200 algas, por lo cual este herbario es el más importante de México.

Contiene alrededor de 650 tipos y las colecciones particularmente valiosas son las de Pringle, Rovirosa, Conzatti, Matuda y Miranda. Se cuenta además con un jardín botánico de aproximadamente 9,000 ejemplares y con una xiloteca de 3000 muestras. Se tiene intercambio con más de 25 herbarios mexicanos y extranjeros. Este herbario tiene como principal finalidad el servir de base para el conocimiento de los recursos florísticos del país.

Gracias a las investigaciones de Helia Bravo se cuenta con su valiosa colección de Cactáceas, mientras que los estudios de Maximino Martínez contribuyeron a formar importantes colecciones de los géneros Pinus y Quercus.

Entre las principales líneas actuales de investigación destacan la taxonomía y ecología de Leguminosas, Cactáceas y Pteridofitas, así como estudios florísticos-ecológicos de los estados de Veracruz y Guerrero.

Cabe mencionar la importancia del trabajo de investigación sobre la Flora de Veracruz dirigido por Arturo Gómez Pompa en colaboración con la Universidad de Harvard, que dará gran impulso al conocimiento de las plantas de ese Estado. La Flora de Veracruz pretende utilizar en sus investigaciones los métodos científicos y técnicos más avanzados, encontrándose acutalmente al. gunos de los investigadores de la institución trabajando en estos estudios.

La institución tiene en la actualidad 23 investigadores y se cuenta con una biblioteca de aproximarlamente 6,700 volúmenes, comprendiendo libros y re- 


\section{HERBARIOS EN MEXICO}

vistas periódicas, además de 1,000 volúmenes pertenecientes a la biblioteca de la Sociedad Botánica de México.

Un resumen histórico del desenvolvimiento de este herbario puede hallarse en las pp. 34, y 35 .

\section{Herbario de la Escuela Nacional de Ciencias Biológicas del Instituto Politécnico Nacional}

El Herbario de la Escuela Nacional de Ciencias Biológicas (siglas ENCB) nació en 1943 bajo la iniciativa del ilustre maestro Maximino Martínez, secundado por Jerzy Rzedowski a partir de 1962. Con inquebrantable voluntad y gran espíritu de lucha logró este infatigable investigador en sólo 10 años colocar al Herbario en un lugar digno, con la ayuda de sus colaboradores Roberto Cruz, Graciela Calderón de Rzedowski, Judith Espinosa y otros en Fanerogamia, así como Gastón Guzmán, Laura Huerta y Ma. Elena Sánchez de Cruz y otros en Criptogamia.

En la actualidad cuenta con una colección de 113,000 fanerógamas, 21,000 hongos, líquenes y mixomixetos, 6,000 algas, 3,500 pteridofitas y 500 briofitas, lo que hace un total de 144,000 ejemplares, más 40,000 que aún no se han identificado o están en proceso de incorporación.

La colección de hongos, líquenes y algas es la más completa del país y quizá de América Latina. Entre las plantas vasculares las mejor representadas son las Gramíneas, Compuestas, Burseráceas, Fagáceas y Pináceas. Además se tiene una colección de polen, semillas, frutos y fósiles.

Tiene un alcance geográfico que comprende todo el país, con particular énfasis en cl Valle de México, cuyas colectas representan cerca de la cuarta parte del herbario. El 5\% coresponde a plantas del extranjero.

Como una de sus funciones se tiene la enseñanza a alumnos de la carrera de biólogo, contándose para esto con pequeño herbario especial. Los alumnos que realizan tesis profesional tienen abierto el herbario general.

La principal función del herbario es la investigación sobre la flora y vegetación. El personal está constituido por 10 investigadores, 1 colector, 3 secretarias, 5 preparadores y 3 o 4 alumnos.

Se tiene intercambio con 8 instituciones nacionales, 5 de Centroamérica y Caribe, 3 de Sudamérica, 17 de Estados Unidos y 5 de Europa. Se despachan en calidad de préstamo 25 a 30 envíos por año. 
Se realiza servicio de identificación de plantas a diversas instituciones y personas, en lo cual se invierte mucha labor, difícil de cuantificar.

Los principales proyectos de investigación ligados a este herbario son: flora y vegetación del Valle de México, flora genérica de México, flora marina de las costas mexicanas, estudios de hongos y líquenes de México y estudios palinológicos.

\section{Herbario del Instituto Nacional de Investigaciones Forestales}

Este herbario tiene como sigla las letras IGF e INIF. Fue fundado en el año de 1958 por Eizi Matuda, habiendo tenido como principales continuadores a Jesús Vázquez Soto, Javier Madrigal Sánchez y Luciano Vela Gálvez.

En la actualidad tiene un acervo aproximado de 25,000 ejemplares, que se espera incrementará proximamente con 5,000 ejemplares más procedentes de los estados de Veracruz, Tabasco y Oaxaca. Como colecciones especiales se tiene una xiloteca, un herbario fitopatológico forestal y un arboretum de coníferas.

Los grupos taxonómicos mejor representados son las coníferas y los árboles de las zonas cálido-húmedas. Su alcance geográfico comprende todo el país y en especial el Eje Neovolcánico.

Dispone de un personal integrado por 6 investigadorees y 4 técnicos.

Como principales funciones se tienen la investigación de los recursos florísticos de las zonas boscosas, consulta nacional y extranjera, pero no se realiza labor de identificación para otros herbarios.

Se mantiene intercambios con tres herbarios nacionales.

Como proyectos hacia el futuro se pretende lograr la mecanización de la información, ampliar el número de ejemplares, no sólo de árboles, sino también de los demás componentes de las comunidades vegetales, para derivar de ahí estudios de caracterización florística de los bosques, así como también realizar estudios biosistemáticos de especies de árboles de importancia forestal.

\section{Herbario de la Comisión Botánica Exploradora del Estado de México, de Toluca, Méx.}

El Herbario de la Comisión Botánica Exploradora, dependiente del Golierno del Estado de México, fue fundado hace 15 años por Eizi Matuda, quien junto con Maximino Martínez, ya fallecido, ha sido su principal impulsor. 


\section{HERBARIOS EN MEXICO}

Cuenta con un acervo de aproximadamente 10,000 ejemplares que representan principalmente la flora del Estado de México. Se dispone de una biblioteca especializada y sus ingresos económicos son proporcionados por el Gobierno del Estado de México.

Fue fundado con el fin de realizar una investigación sobre la flora deI Estado de México, aunque también tiene entre sus finalidades el atender consultas de estudiantes y del público en general.

Los resultados de las investigacionese se han publicado en 20 números del Boletín de la Comisión Botánica, así como también en otras revistas.

\section{Herbario de la Facultad de Ciencias Biológicas de la Universidad Autónoma de Nuevo León}

El Herbario de la Facultad de Ciencias Biológicas de la Universidad Autónoma de Nuevo León, en Monterrey, fue fundado en el año de 1942 por Antonio Hernández Corzo. Su colección está constituida por 12,200 ejemplares de fanerógamas, 3,500 de hongos, 2,700 de algas, y 2,000 de otras criptógamas, haciendo un total de 20,400 ejemplares.

$\mathrm{Su}$ alcance geográfico comprende todo el país, pero especialmente los estados de Nuevo León, Veracruz y San Luis Potosí. Los grupos mejor representados son los hongos y las algas.

Entre sus principales finalidades figura la enseñanza, para la cual se tiene un herbario especial constituido por 2,000 ejemplares. Se realiza investigación sobre los recursos florísticos de Nuevo León y Veracruz.

El personal está constituido por tres investigadores y cuatro alumnos que ayudan en el trabajo del herbario.

Se realiza intercambio con cuatro herbarios nacionales y tres extranjeros. Como colección especial se tiene una xiloteca de 110 ejemplares.

Entre los principales problemas que afronta el herbario figuran la carencia de una biblioteca especializada, de personal técnico y de un presupuesto propio.

\section{Herbario del Instituto Tecnológico y de Estudios Superiores de Monterrey}

El Herbario del Instituto Tecnológico y de Estudios Superiores de Monterrey tiene 20 años de haber sido fundado como una dependencia de la Escuela de Agronomía, con fines de enseñanza. En la actualidad forma parte del Departamento de Biología del mismo Instituto. Sus siglas son MEMO. 
El acervo de especímenes es relativamente pequeño, con un número aproximado de 2,500 que pertenecen a plantas vasculares, incluyendo algunas pteridofitas, y representando principalmente la flora del NE de México. Sin embargo, hay ejemplares de otras zonas del país, así como también del extranjero. La familia de las Gramíneas es la mejor representada.

Este herbario carece de presupuesto propio, personal científico y técnico, así como también de una biblioteca especializada. Esta es la razón de que el número de especímenes no se incremente.

Por otra parte existe el proyecto de aumentar el número de ejemplares e iniciar una colección fitopatológica.

VII. Herburio del Instituto de Investigación de Zonas Desérticas de la Universidad Autónoma de San Luis Potosí.

El Herbario del Instituto de Investigación de Zonas Desérticas ha recibido el nombre de Herbario Isidro Palacios. Ha sido fundado en 1954 por Jerzy Rzedowski, quien fue su director y principal impulsor durante sus primeros años.

Su director actual es Fernando Medellín Leal, quien tiene como colaboradores a Fernando Gómez Lorence y a Sonia Nelly Salas de León.

El número de ejemplares asciende a aproximadamente 7,500, representando principalmente la flora fanerogámica de las zonas áridas de México y en especial del c:tado de San Luis Potosí.

Fue fundado con fines de investigación, habiéndose publicado varios trabajos de tesis e investigación en Acta Científica Potosina y en otras revistas nacionales y extranjeras. Las colecciones más importantes con que cuenta este herbario son las de J. Rzedowski y J. G. Schaffner.

El Herhario Isidro Palacios realiza intercambio y préstamos de material botínico con herbarios mexicanos y del extranjero. Luenta además con una mo desia biblioteca especializada.

VIIT. Horbario de la Escuela de Biología de la Universidad Veracruzana, en Balapa.

E. Herbario de la Escuela de Biología de la Universidad Veracruzana fue fundado on 3971 con fines de enscinanza, siendo su fundador y principal impulsor Mario Vázquez Torres, quien ha contado con la ayuda de Jesís l)orantes. 
En la actualidad tiene un número aproximado de 5,000 ejemplares, principalmente angiospermas y macromicetos. La mayoría de los ejemplares son representativos de la flora del estado de Veracruz.

El material ha sido colectado por el personal adscrito al herbario, formado por dos botánicos, profesores de la Escuela, y seis alumnos de la misma. Algunos de los ejemplares provienen de intercambio con otros herbarios nacionales.

Entre los problemas que impiden el desarrollo de este herbario se tienen: la falta de un local y mobiliario adecuado, carencia de un presupuesto propio y de una biblioteca especializada.

El personal que labora en él se ha propuesto resolver pronto estas deficiencias y desea alcanzar la cifra de 50,000 ejemplares en los próximos cuatro años.

IX. Herbario de la Escuela de Agricultura de la Universidad de Guadalajara.

El Herbario de la Escuela de Agricultura de la Universidad de Guadalajara fue fundado en el año de 1960 por Luz María Villarreal de Puga, de quien ha recibido gran impulso.

En la actualidad tiene un acervo de 25,000 ejemplares, de los cuales 15,000 no se han identificado ni registrado. Existe una colección de 5,000 ejemplares que se utiliza para la enseñanza a los alumnos de la carrera de Ingeniero Agrónomo. Tiene como principal finalidad la investigación de la flora y vegetación de la zona llamada Nueva Galicia, principalmente los estados de Jalisco, Colima, Michoacán y Zacatecas.

Los grupos taxonómicos mejor representados pertenecen a las familias Leguminosae, Compositae y Gramineae. Se cuenta con una colección especial de 800 cjemplares, donada por Rodolfo Meza, correspondiente a la flora del estado de Aguascalientes. Además se tiene un invernadero con helechos y cactáceas de Jalisco.

El personal está constituido por un investigador y un preparador. Tiene asignada una partida modesta del presupuesto general de la Universidad de Guadalajara. Cuenta con una biblioteca que se considera muy deficiente.

Se tiene intercambio con dos herbarios nacionales y uno extranjero. Se pretende para el futuro levantar inventario de las gramíneas del estado de Jalisco, además de publicar un boletín periódico sobre la flora del Valle de Guadalajara. 


\section{Herbario de la Escuela de Biología de la Universidad Autónoma de Gua- dalajara.}

El Herbario de la Escuela de Biología de la Universidad Autónoma de Guadalajara fue fundado en el año de 1967 por Carlos Luis Díaz Luna, quien junto con David García Saucedo han sido sus principales impulsores.

En la actualidad tiene un acervo de 7,400 ejemplares. De éstos 5,000 son fanerógamas, 1,500 hongos, 300 pteridofitas, 250 algas, 200 briofitas y 150 líquenes.

Tiene un área de acción que comprende los estados de Jalisco Colima, Zacatecas, Michoacán y Nayarit. Además, se han hecho importantes colectas en los estados de San Luis Potosí, Sinaloa, Sonora y Baja California.

Entre sus finalidades se tiene la enseñanza a alumnos de la carrera de biólogo, para lo cual se dispone de un pequeño herbario de 1,000 ejemplares. Otro de sus objetivos es realizar una investigación de la flora y de la vegetación de la zona.

El personal está constituido por dos investigadores, un preparador y una secretaria. Se cuenta con una biblioteca muy modesta, con 600 volúmenes, incluyendo libros y revistas. El financiamiento depende del presupuesto de la Escuela de Biología de la Universidad.

Se realizan intercambios con cuatro herbarios nacionales y cinco extranjeros.

Se cuenta con una colección especial fitopatológica de 300 hongos parásitos y una xiloteca con 200 ejemplares. En el año de 1968 se fundó un jardín botánico que cuenta con 8,000 ejemplares, principalmente cactáceas y suculentas. Se dispone de un almácigo y de un invernadero para especies tropicales. El personal del jardín botánico está constituido por dos investigadores y quince jardineros.

Se pretende en el futuro realizar una investigación sobre la flora y la vegetación de la Laguna de Cuyutlán, Colima; flora y vegetación del Valle de Guadalajara, el estudio de los macromicetos y de briofitas del estado de Jalisco, así como de plantas arvenses del Valle de Guadalajara y una investigación palinológica de las Leguminosas de Jalisco.

En resumen, la historia y el estado actual de los herbarios de México revelan que estas instituciones han tenido en general poco arraigo y tradición en el país y no es sino en los últimos 30 años cuando comienza a verse una tendencia francamente ascendente en el incremento substancial tanto del número de los herbarios como de sus acervos. 


\section{HERBARIOS EN MEXICO}

Queda aún mucho trecho por recorrer sólo para lograr que las colecciones nacionales alcancen un nivel comparable con muchos herbarios extranjeros, con lo cual se sentarían las bases para la realización de muchas investigaciones que hoy no se pueden hacer en el país. Es importante asimismo que los herbarios se diversifiquen en función de la demanda y que se aumente el número de disciplinas que son objeto de estudio en ellos.

Sería de desearse que las investigaciones que se realizan en los herbarios mexicanos estén orientadas hacia el mejor aprovechamiento y conservación de nuestros recursos bióticos creando conciencia nacional en el pueblo, autoridades e instituciones educativas de que se debe incrementar la labor de los herbarios y por ello es preciso dar a conocer la importancia que tiene esta tarea para la economía del país. 